\title{
Reducing medication error and polypharmacy in older people
}

\author{
Nadzirah Rosli, MBChB, Shyh Poh Teo, MBChB, FRACP
}

\begin{abstract}
Taking multiple medications for the treatment of chronic diseases may lead to medication-related problems or adverse effects. Physiological changes with age affect drug absorption and clearance and hence have implications on drug doses. Hospitalised older people are at risk of prescribing errors. Medication reconciliation should be performed at all interfaces of care, including admission, transfer, and discharge. Polypharmacy and inappropriate medications are also common in older people, for whom deprescribing should be initiated, taking into account their life expectancy and applying a holistic approach to achieve individualised care.
\end{abstract}

Key words: Aged; Deprescriptions; Drugs; Drug-related side effects and adverse reactions; Polypharmacy
Geriatrics and Palliative Unit, Raja Isteri Pengiran Anak Saleha Hospital, Brunei Darussalam

Correspondence to: Dr Shyh Poh Teo, Geriatrics and Palliative Unit, Raja Isteri Pengiran Anak Saleha Hospital, Jalan Putera Al-Muhtadee Billah, Bandar Seri Begawan, BA1710, Brunei Darussalam. Email: shyhpoh.teo@moh.gov.bn

\section{INTRODUCTION}

In the United States, people aged 65 years and older constitute $13 \%$ of the population and purchase $33 \%$ of all prescription medications. ${ }^{1}$ By 2040 , older people are expected to make up $25 \%$ of the population and to purchase $50 \%$ of all prescription drugs. ${ }^{2}$

For geriatric medicine, medications are prescribed to treat chronic disease, alleviate pain, and improve quality of life. However, the increased use of medications is associated with the risk of medication-related problems or adverse effects. ${ }^{3}$ Clinicians should be aware of the effects of ageing physiology on drug therapy, taking into account age-related changes in pharmacokinetics and pharmacodynamics before prescribing medications. ${ }^{4}$

In this review, important considerations in prescribing medications for older people are discussed, focusing on ageing physiology and the impact on drugs, reducing prescribing error through medication reconciliation and deprescribing for polypharmacy.

\section{AGEING PHYSIOLOGY AND IMPACT ON DRUGS}

The ageing process may affect drug absorption and metabolism and may have implications for drug prescribing. Dysphagia may affect the ability to swallow tablets. Medications may need to be crushed or prepared as liquid formulations. However, it is important to recognise the consequences of manipulating medicines by splitting or crushing. This may alter absorption characteristics, resulting in local irritant effects, unacceptable taste, and medication instability. Controlled or slow-release medications tend to lose these properties when crushed and should be administered in divided doses.

Once ingested, changes in absorption and slow gastric emptying may result in a lower or delayed peak concentration. A reduction in hepatic blood flow or size causes reduced hepatic microsomal drug activity and reduced hepatic metabolism. First pass metabolism is also reduced, resulting in increased bioavailability of medications such as warfarin, fentanyl, and amiodarone. The reduced number of 
functioning nephrons, renal blood flow, and tubular secretion also affects the renal clearance of drugs. This has implications on renally cleared medications, such as lithium and digoxin, where levels and close monitoring of adverse effects are required. Decreased drug clearance may need dose-adjustments, depending on estimated function of the hepatic and renal organs in older people. The relative amount of water in the body and volume of distribution reduces, hence fat-soluble drugs tend to remain longer in the body with prolonged effects, with implications for sedatives and anxiolytics, including benzodiazepines. Drug toxicity may only gradually develop over time, as concentrations increase after about four half-lives to reach a steady state. ${ }^{4}$

Pharmacodynamics of drugs should also be considered. The central nervous system is a vulnerable drug target in older people. Between 20 to 80 years of age, cerebral blood flow reduces by $30 \%$, weight by $20 \%$, with a $30 \%$ decrease in cortical neuronal density. ${ }^{5}$ Thus, it is important to be cautious with medications that act on the nervous system. For example, reduced dopamine may place older people at risk of extrapyramidal symptoms with dopamine antagonists such as metoclopramide, whereas reduced acetylcholine may increase problems of anticholinergic effects with neuroleptics and tricyclic antidepressants. ${ }^{6}$

Compliance to medications should be checked. For those who are cognitively impaired, pill boxes, reminders, and reduction of dose frequency may be helpful. Patients with reduced dexterity or arthritis may be unable to open small packets used to dispense or store medications. It is also important to assess inhaler technique, including spacer use for older people who are prescribed these devices.

\section{REDUCING PRESCRIBING ERROR THROUGH MEDICATION RECONCILIATION}

In addition to taking ageing physiology into account, medication reconciliation is important to reduce prescribing errors for patients admitted to hospital. Prescribing errors usually occur during hospital admissions, with $10 \%$ to $67 \%$ of medication histories having at least one error, and up to $33 \%$ of these errors potentially causing harm. Patients with one or more medicines missing from their discharge information are 2.3 times more likely to be readmitted to hospital than those with correct information on discharge. ${ }^{7}$ More than $50 \%$ of medication errors occur at transitions of care, with $85 \%$ of these discrepancies occurring owing to poor medication history taking. ${ }^{8}$

Medication reconciliation aims to check the accuracy of drugs prescribed. When a patient is transferred to another clinician, a current and accurate medication list, including changes made, should be handed over. However, prescribing errors may occur during hospital admissions, transfers between emergency departments, intensive care and other wards, inter-hospital transfers, or discharge from hospital. Medication reconciliation can reduce prescribing error, particularly at all interfaces of care (admission, transfer, and discharge), through eliminating undocumented intentional or unintentional discrepancies. ${ }^{7}$

An example of an undocumented intentional discrepancy is as follows: a patient admitted for elective surgery was not prescribed antihypertensive medication owing to concerns regarding perioperative hypotension. On discharge, this medication was not prescribed. If the patient was not informed regarding the change, they may enquire with their family doctor, surgeon, or pharmacist. However, the rationale may not be identified without documentation regarding the change. In an unintentional discrepancy, a patient admitted for elective surgery was noticed as 'unmotivated' after surgery, and refused to mobilise. Because antidepressant medication normally taken before admission had not been prescribed in hospital, the delayed onset of action resulted in prolonged hospitalisation. These scenarios illustrate the risks of prescribing errors resulting in time wasted to check on discrepancies, safety issues, morbidity, and prolonged hospitalisation.

Several errors may be prevented through medication reconciliation. This includes failure to prescribe clinically important medications from home, incorrect doses or dosage forms, missed or duplicate doses due to inaccurate records, failure to specify which medications to resume or discontinue after hospital discharge, or duplicate therapy at discharge, usually due to brand or generic name combinations or hospital formulary substitutions. ${ }^{9,10}$ 
Medication reconciliation consists of the following steps: firstly, a list of current medications should be obtained, followed by a list of medications needed during the admission. Both lists should be compared, and a new list generated based on the comparison. This new list should be communicated to the patient and caregivers to avoid confusion. ${ }^{11}$

It is useful to ask patients to bring in all their medications taken at home, including complementary or alternative medicines and supplements. This helps identify all drugs they consume, including from different hospitals or private clinics, even if they are absent from the hospital records. Cessation of drugs should be documented and emphasised, with consideration of removing home supplies of discontinued drugs. For patients who had multiple readmissions, it is worthwhile to check if patients still need a prescription for regular medications before discharge; rather than automatically offering another to avoid cumulating drugs at home. ${ }^{12}$

\section{POLYPHARMACY AND DEPRESCRIBING}

In the United States, $40 \%$ of people aged 65 years and older take 5 to 9 medications, with $18 \%$ taking ten or more medications. ${ }^{13}$ In the United Kingdom, the average number of medications prescribed to older adults doubled from 21.2 to 40.8 items per year between 1997 and 2006. ${ }^{14}$ Polypharmacy increases the risk of adverse drug events. The risk of adverse drug events increases from 13\% with two medications to $58 \%$ with 5 medications and $82 \%$ with $>7$ medications. ${ }^{15}$ Older adults are also 7 times more likely than younger people to have adverse drug events requiring hospitalisation. ${ }^{16}$

Although there is no consensus regarding how many medications constitutes polypharmacy, the generally accepted cut-off is five or more medications. Polypharmacy can also be determined on the basis of appropriateness, rather than absolute numbers. This defines polypharmacy as the administration of more medications than are clinically indicated, representing unnecessary use.

For older people with multiple comorbidities, clinicians prescribe medications to achieve guideline-based care. Adverse drug reactions may go unrecognised, resulting in a prescribing cascade, where more medications are prescribed to manage symptoms caused by other medications. Patients are thus at risk of adverse effects related to potentially unnecessary treatment. Therefore, before new medications are started, clinicians should consider non-pharmacological management first, and then starting at the lowest feasible drug dose with a preference for those with lower risk of adverse effects. ${ }^{17}$

Occasionally medications are started in hospital as short-term treatment. However, these may be continued indefinitely, as there were no specific instructions on duration of treatment. Common medications include antipsychotics for delirium or insomnia, and concurrent proton pump inhibitors with non-steroidal anti-inflammatory drugs. ${ }^{14,16}$

The management approach for polypharmacy is deprescribing. Deprescribing is the 'systematic process of identifying and discontinuing drugs in instances in which existing or potential harms outweigh existing or potential benefits within the context of an individual patient's care goals, current level of functioning, life expectancy, values, and preferences ${ }^{\prime}{ }^{18}$ Clinicians should consider a patient's possible life expectancy with the time to benefit from medications. If the suspected life expectancy is likely less than the time to benefit, the medication is generally not recommended. In applying this concept, prevention should be focused on healthier older adults, whereas symptom-oriented treatment should predominate in sicker or frail older adults. ${ }^{19}$ Hence, in addition to polypharmacy, deprescribing should also be considered when there are adverse drug reactions or drug interactions, non-compliance, reduced benefit or effectiveness, indications of shortened life expectancy, terminal illness, dementia or frailty. ${ }^{20}$

Deprescribing is a process that should start when a medication is prescribed, particularly for short-term indications. The patient should be informed regarding duration of treatment at the outset. When adverse drug reactions occur, clinicians should recognise these complications and prioritise medications to discontinue. Some medications should be gradually weaned off due to risk of rebound or withdrawal, such as opioids, benzodiazepines, steroids, and beta-blockers. Patients should also be monitored to ensure there were no complications from medication 
cessation. ${ }^{21}$ Thus, deprescribing medications can be prioritised as follows: stop medications not taken by the patient, medications likely to cause adverse effects or harm, and those less likely to benefit the patient. ${ }^{18,21}$

Deprescribing can be challenging and met with resistance from patients or family members, clinicians' reluctance to discontinue medications from other clinicians and fear of drug withdrawalrelated adverse effects. However, deprescribing has been shown to be beneficial by improving patient medication knowledge and adherence, resolution of adverse drug reactions, reduced financial costs and mortality, particularly in those aged 65 to 80 years. ${ }^{18,20}$

A prospective study reported the outcomes of comprehensive geriatric assessment and deprescribing by geriatricians for older community patients with polypharmacy. Deprescribing is welltolerated and associated with improved clinical outcomes in terms of functional and cognitive status, appetite, sleep, and reductions in major complications. These benefits occur within 3 months of the intervention, persisting for $>2$ years in more than two-thirds of the patients. ${ }^{22}$ A cluster randomised controlled trial found that deprescribing by geriatricians and pharmacists in Dutch nursing homes was more likely to result in discontinuation of at least one inappropriate medication without any deterioration in patient well-being. ${ }^{23}$

Two useful tools to indicate inappropriate medications in older people are the Screening Tool of Older Persons' Prescriptions (STOPP) criteria and Beer's criteria. ${ }^{24,25}$ STOPP is the preferred tool, as it identified more inappropriate medications that led to adverse events and hospitalisation compared to Beer's criteria. ${ }^{26,27}$ Examples of inappropriate medications listed include metoclopramide for patients with parkinsonism, loop diuretics for dependent ankle oedema only without clinical signs of cardiac failure, treatment dose proton pump inhibitor for peptic ulcer disease for $>8$ weeks, and calcium-channel blockers for those with chronic constipation. However, both tools do not take into account the risk-benefit ratio of medications. The benefits of medications may vary from randomised controlled trials due to co-existing conditions, thus further research is required to clarify treatment effects for patients with multiple co-morbidities. ${ }^{28}$
Overall, deprescribing should involve discontinuing inappropriate medications particularly those causing adverse effects, taking into account patient life expectancy and applying a holistic approach to achieve individualised goals of care. Further work is required to consolidate the medication review process into a systematic algorithm incorporating deprescribing of potentially inappropriate medications, a scoring scheme to quantify the medication review process, and practically applying these concepts into clinical practice. ${ }^{29}$

\section{AN ILLUSTRATIVE CASE}

An 88-year-old woman presented with a 1-week history of fever and coughing up white sputum while eating. She had a history of congestive cardiac failure and multiple falls, with a previous subtrochanteric fracture repaired with intramedullary nailing 10 years prior. She also had a right middle cerebral artery infarct 5 years prior with residual hemiplegia, cognitive impairment, and suspected vascular dementia. She was wheelchair-bound and required assistance for transfer and activities of daily living. Her medications included aspirin, atorvastatin, amlodipine, furosemide, allopurinol, alendronic acid, omeprazole, multivitamins, salbutamol, and fluticasone and salmeterol inhalers. Clinical positive findings were mild hypoxia, bibasal coarse crackles, and expiratory wheeze. Blood test results revealed raised inflammatory markers with normal hepatic and renal function. She was treated for communityacquired pneumonia with intravenous injection of co-amoxiclav, which was changed to oral tablets after 48 hours. Assessment by a speech and language therapist revealed that she could take a normal diet and swallow medication tablets. On discharge from the hospital, she was prescribed a short course of oral co-amoxiclav, omeprazole was gradually downtitrated, and atorvastatin, furosemide, alendronic acid, and multivitamins were discontinued.

The patient's vascular dementia and increased dependence suggest that it may be inappropriate to prescribe multiple preventive drugs such as bisphosphonates and statins. For the remaining essential medications, it is important to ensure that they can be taken appropriately and safely. Given her neurological impairment and history suggestive of aspiration, her swallowing was assessed. As family 
members assisted with medicine administration, their inhaler technique using a spacer was reviewed before discharge. Follow-up was organised, with advice given to seek medical attention early should symptoms of cardiac failure occur due to rebound effects.

\section{CONCLUSION}

Due to physiological changes with ageing, dose adjustments may be required for older people to avoid drug toxicity. Medication reconciliation is important to reduce prescribing errors, which are common during transfer of care, particularly hospital admissions or discharge. Older people are also prone to polypharmacy and adverse drug reactions, which should prompt clinicians to initiate deprescribing.

\section{DECLARATION}

The authors have no conflicts of interests to disclose. No funds were obtained for production of the manuscript.

\section{REFERENCES}

1. Charlesworth CJ, Smit E, Lee DS, Alramadhan F, Odden MC. Polypharmacy among adults aged 65 years and older in the United States: 1988-2010. J Gerontol A Biol Sci Med Sci 2015;70:98995. Crossref

2. Reddy P, Gosavi D, Varma SK. An overview of geriatric pharmacology. Asian J Pharmaceut Clin Res 2012;5:25-9.

3. Lavan AH, Gallagher P. Predicting risk of adverse drug reactions in older adults. Ther Adv Drug Saf 2016;7:11-22. Crossref

4. Mangoni AA, Jackson SH. Age-related changes in pharmacokinetics and pharmacodynamics: basic principles and practical applications. Br J Clin Pharmacol 2004;57:6-14. Crossref

5. Knight J, Nigam Y. Exploring the anatomy and physiology of ageing. Part 5: the nervous system. Nurs Times 2008;104:18-9.

6. Grahame-Smith DG, Aronson JK. The Oxford Textbook of Clinical Pharmacology and Drug Therapy. 3rd ed. The drug history and the clinical examination and investigation of drug effects. Oxford: Oxford University Press; 2002: 167-70. Crossref

7. Kreckman J, Wasey W, Wise S, Stevens T, Millburg L, Jaeger C. Improving medication reconciliation at hospital admission, discharge and ambulatory care through a transition of care team. BMJ Open Qual 2018;7:e000281. Crossref

8. Fitzgerald RJ. Medication errors: the importance of an accurate drug history. Br J Clin Pharmacol 2009;67:671-5. Crossref

9. Kanjanarat $P$, Winterstein AG, Johns TE, Hatton RC, Gonzalez-Rothi R, Segal R. Nature of preventable adverse drug events in hospitals: a literature review. Am J Health Syst Pharm 2003;60:1750-9. Crossref

10. Cornish PL, Knowles SR, Marchesano R, et al. Unintended medication discrepancies at the time of hospital admission. Arch
Intern Med 2005;165:424-9. Crossref

11. Gleason KM, Groszek JM, Sullivan C, Rooney D, Barnard C, Noskin GA. Reconciliation of discrepancies in medication histories and admission orders of newly hospitalized patients. Am J Health Syst Pharm 2004;61:1689-95. Crossref

12. SP Teo. Sensible prescribing for older adults: illustrated cases. J Gerontol Geriatr 2017;65:130-3.

13. Murray MD, Kroenke K. Polypharmacy and medication adherence: small steps on a long road. J Gen Intern Med 2001;16:137-9. Crossref

14. Patterson SM, Cadogan CA, Kerse N, et al. Interventions to improve the appropriate use of polypharmacy for older people. Cochrane Database Syst Rev 2014;10:CD008165. Crossref

15. Budnitz DS, Lovegrove MC, Shehab N, Richards CL. Emergency hospitalizations for adverse drug events in older Americans. N Engl J Med 2011;365:2002-12. Crossref

16. Johansson $\mathrm{T}$, Abuzahra ME, Keller $\mathrm{S}$, et al. Impact of strategies to reduce polypharmacy on clinically relevant endpoints: a systematic review and meta-analysis. Br J Clin Pharmacol 2016;82:532-48. Crossref

17. Rochon PA, Gurwitz JH. Optimising drug treatment for elderly people: the prescribing cascade. BMJ 1997;315:1096-9. Crossref

18. Scott IA, Hilmer SN, Reeve E, et al. Reducing inappropriate polypharmacy: the process of deprescribing. JAMA Intern Med 2015;175:827-34. Crossref

19. Lee SJ, Kim CM. Individualizing prevention for older adults. J Am Geriatr Soc 2018;66:229-34. Crossref

20. Bain KT, Holmes HM, Beers MH, Maio V, Handler SM, Pauker SG. Discontinuing medications: a novel approach for revising the prescribing stage of the medication-use process. J Am Geriatr Soc 2008;56:1946-52. Crossref

21. Le Couteur D, Gnjidic D, McLachlan A. Deprescribing. Aust Prescr 2011;34:182-5. Crossref

22. Garfinkel D. Poly-de-prescribing to treat polypharmacy: efficacy and safety. Ther Adv Drug Saf 2018;9:25-43. Crossref

23. Wouters $\mathrm{H}$, Scheper J, Koning $\mathrm{H}$, et al. Discontinuing inappropriate medication use in nursing home residents: a cluster randomized controlled trial. Ann Intern Med 2017;167:609-17. Crossref

24. Gallagher P, Ryan C, Byrne S, Kennedy J, O'Mahony D. STOPP (Screening Tool of Older Persons' Prescriptions) and START (Screening Tool to Alert Doctors to Right Treatment): Consensus validation. Int J Clin Pharmacol Ther 2008;46:72-83. Crossref

25. American Geriatrics Society 2015 Beers Criteria Update Expert Panel. American Geriatrics Society 2015 updated Beers criteria for potentially inappropriate medication use in older adults. J Am Geriatr Soc 2015;63:2227-46. Crossref

26. Gallagher P, O'Mahony D. STOPP (Screening Tool of Older Persons' potentially inappropriate Prescriptions): application to acutely ill elderly patients and comparison with Beers' criteria. Age Ageing 2008;37:673-9. Crossref

27. Ubeda A, Ferrandiz L, Maicas N, Gomez C, Bonet M, Peris JE. Potentially inappropriate prescribing in institutionalised older patients in Spain: the STOPP-START criteria compared with the Beers criteria. Pharm Pract (Granada) 2012;10:83-91. Crossref

28. Tinetti ME, McAvay G, Trentalange M, Cohen AB, Allore HG. Association between guideline recommended drugs and death in older adults with multiple chronic conditions: population based cohort study. BMJ 2015;351:h4984. Crossref

29. Thiruchelvam K, Wong PS, Kairuz T, Babar ZU, Hasan SS. Consolidated medication review algorithm to improve medications use in older adults: components, scoring scheme, and implementation. J Am Med Dir Assoc 2018;19:7178. Crossref 